\title{
Performance Analysis of Satellite Communication Systems with Randomly Located Ground Users
}

\author{
Dong-Hyoun Na, Student Member, IEEE, Ki-Hong Park, Senior Member, IEEE, Young-Chai Ko, Senior \\ Member, IEEE, and Mohamed-Slim Alouini, Fellow, IEEE
}

\begin{abstract}
Satellite communication (SatCom) is an essential component of next-generation wireless communication to achieve a goal of ubiquitous connectivity on globe. The outage event of SatCom link connecting to a network is more critical in an infrastructure-deficient remote area. In this paper, we analyze outage probability (OP) and symbol error rate (SER) over SatCom downlink channels when the users are randomly located in single beam and multibeam area. The downlink beams will suffer from propagation loss and the shadowed-Rician fading depending on the user location which is assumed to follow a Poisson point process. For mathematically tractable, informative, and insightful interpretation, we obtain the asymptotic $O P$ and SER expressions of user link under several channel conditions in the high power regime. Finally, numerical results are presented to verify the analysis and show the accuracy of the asymptotic results.
\end{abstract}

Index Terms-Satellite systems, shadowed-Rician, stochastic geometry, Poisson point process, outage probability, symbol error rate.

\section{INTRODUCTION}

$\mathbf{S}$ ATELLITE communications (SatCom) offer an appealing solution for rural connectivity owing to their almost ubiquitous coverage and high throughput. For the same reasons, they are crucial for enabling many critical applications, such as maritime and aviation communications [1]. In order to provide against the unprecedented demand on data and densification in $5 \mathrm{G}$ network, SatCom systems have recently attracted significant research attention [2]. Beyond 5G, SatCom systems have been also considered to apply to $6 \mathrm{G}$ network in order to guarantee communication fairness since SatCom can provide access for rural areas and achieve the worldwide connectivity [3], [4]. In addition, SatCom systems realize non-terrestrial communication (NTC), a key component in 6G. Recently, the authors in [5] investigated recent advances in NTC and calculated the performance of the NTC scenario utilizing satellites.

Manuscript received Month XX, XXXX; revised Month XX, XXXX; accepted Month XX, XXXX. Date of publication Month XX, XXXX; date of current version Month XX, XXXX. This research was funded in part by KAUST Office of Sponsored Research and in part by Institute of Information \& Communications Technology Planning \& Evaluation (IITP) grant funded by the Korea government (MSIT) (2021-0-00260, Research on LEO Inter-Satellite Links).

D.-H. Na and Y.-C. Ko are with the School of Electrical Engineering, Korea University, Seoul 02841, South Korea (e-mail: ab2192@korea.ac.kr; koyc@korea.ac.kr).

D.-H. Na, K.-H. Park, and M.-S. Alouini are with the Computer, Electrical, and Mathematical Sciences and Engineering Division, King Abdullah University of Science and Technology, Thuwal 23955, Saudi Arabia (e-mail: donghyoun.na@kaust.edu.sa; kihong.park@kaust.edu.sa; slim.alouini@kaust.edu.sa).
Geosynchronous (GEO) satellites, with orbits at altitudes of about $36,000 \mathrm{~km}$, receive downlink signals from gateways along feeder links, and transmit to ground users via user links. Traditionally, a single beam GEO SatCom system covers wide area without interference due to the strict frequency reuse or spacing. These days, more aggressive frequency reuse among multiple beams has been widely adopted to imitate terrestrial cellular networks for higher data rate. In one multibeam method, four-frequency reuse scheme divides the frequency band into four so that adjacent beams employ different band to make them disjoint [6]. Therefore, inter-beam interference from the surrounding beams can be alleviated. To obtain higher data rate with a limited spectrum band, full frequency reuse scheme is taken into account as a multibeam method, which takes advantage of all the bandwidth available on the user link for all beams [7]. However, inter-beam interference is a critical issue to properly handle in the full frequency reuse scheme.

\section{A. State of the Art Literature}

Interference mitigation techniques such as precoding and beam/cluster hopping methods are required to reduce the inter-beam interference. The inter-beam interference can be controlled by precoding at gateways or on-board processing at satellites through channel feedback [7]-[10]. The problem is that GEO SatCom systems receive an outdated channel state information (CSI) due to the long round-trip delay. If only on-board processing can sufficiently diminish inter-beam interference, the latency due to round-trip delay can be reduced by half. Furthermore, because energy efficiency is an important performance metric to optimize the size and lifetime of the satellite, power allocation through precoding has been studied [11]-[13]. The beam/cluster hopping is another technique of reducing the inter-beam interference by activating only high demand beams or clusters per unit time [14], [15]. It is based on the fact that the demands of the regions covered by each beam are different over time. Since the number of beams exploited at one time decreases, it can not only mitigate interference, but also allocate greater power to the activated beams.

Moreover, if the line-of-sight (LoS) cannot be obtained due to obstacles between the satellite and users, performance can be degraded significantly. To solve this issue and improve the reliability of the link, hybrid satellite-terrestrial relay networks (HSTRNs) have been proposed. HSTRNs are cooperative systems where a satellite transmits signals to destinations with the aid of a relay node located at ground. For example, 
the authors in [16]-[23] analyzed symbol error rate (SER) and ergodic capacity in HSTRNs. In [20], [23], performance analysis was carried out after approximating fading channel, assuming that one of the satellite's channel parameters is an integer or rational number. In [21], [23], outage probability (OP) was analyzed assuming a situation in which a signal is transmitted to a user by selecting one of the multiple relays on the ground. In addition, the performance of a situation using beamforming in HSTRNs with multiple antennas has been studied in [17], [22], [23]. In [24], [25], the concept of cognitive radio was introduced in which satellite and terrestrial networks employ the same resources. The authors in [24] proposed an algorithm that maximizes the rate and uses beamforming to reduce interference from ground users and inter-beam interference. In [25], OP was analyzed by distributing ground users and terrestrial base stations (BSs) with a stochastic geometry. Stochastic geometry is a concept exploited to analyze system performance of randomly distributed users. In [26], coverage and rate performance in cellular networks using millimeter waves were analyzed with stochastic geometry. In [27], [28], the distributions of BSs and users were modeled by PPP, and performance analyses were conducted. Both distances between randomly distributed transceivers and Rayleigh fading were considered, where stochastic geometry was used as a tool. A tutorial on stochastic geometry in cellular networks was presented in [29]. The practicality of stochastic geometry has been demonstrated and a unified approach for performance analysis in various system models was proposed. Analyses using the tool from stochastic geometry have also been carried out in three-dimensional wireless networks with unmanned aerial vehicle (UAV). The authors in [30] considered a network with a finite number of UAVs and a receiver at an arbitrary location. The UAVs were modeled with binomial point process and Nakagami- $m$ fading was assumed to derive downlink coverage probability utilizing stochastic geometry. In [31], UAVs and BSs were modeled as independent PPPs, considering a downlink network where UAVs assist cellular networks by covering hotspot regions. The performance analysis exploiting stochastic geometry in this system has been presented. The authors in [32] analyzed the statistical characteristics of Doppler spread in SatCom NTC situations. In order to improve resource utilization efficiency, the non-orthogonal multiple access (NOMA) scheme has been recently proposed in SatCom systems [33], [34]. With the NOMA scheme, superposed signals in the power domain are transmitted through a frequency band simultaneously, and interference caused by superposition can be eliminated by using successive interference cancellation at the receiver. In [35], OP was analyzed in a situation where two destinations of HSTRNs receive signals from a relay with NOMA. The authors in [36] adopted NOMA to user link of SatCom and investigated its performance such as OP, SER, and ergodic capacity.

\section{B. Motivation and Contributions}

In this paper, we investigate the performance in single beam and multibeam of SatCom downlink channels with a distribution of ground users, where we assume that the multiple users in each beam are randomly located as a Poisson point process (PPP). The motivation and contributions of this paper can be summarized as follows.

- The SatCom channel is affected by several factors, i.e., free space path loss (FSPL), beam pattern, and small scale fading on propagation. In order to analyze the performance in SatCom thoroughly, we must take all the random factors into account. However, the aforementioned works only dealt with one of them in their analysis. For example, only small scale fading was taken into consideration with fixed position of ground users in the previous works [16]-[23], [35], [36]. However, the satellite beam covers ultra wide area where the users can be randomly located and, correspondingly, the beam gain is widely varying over beam coverage. Therefore, we derive the OP and SER performance over the randomness of all the elements in the SatCom channel model which consists of beam pattern, FSPL, and shadowed-Rician (SR) fading.

- In [25], satellite users were uniformly distributed with PPP as in this paper, but it was assumed that there was only one user per beam. Additionally, Gamma random variable was adopted instead of SR fading since it is amenable to analyze than SR fading. However, we consider a situation where there are multiple users per beam and employ SR fading as user link channel model.

- By utilizing some stochastic geometry properties, we obtain a closed form result of the asymptotic OP in the high signal-to-noise ratio (SNR) region. We also derive an asymptotic SER based on the asymptotic cumulative distribution function (CDF).

- Our proposed analytical approach is applied to different user access scenarios, e.g., maximum SNR user selection, multicast scheduling to support multiple users in a group at a user's rate with the minimum SNR, and random selection for fairness. Finally, the numerical results of the analysis for each scenario are presented to validate our analytic results and we also demonstrate that the parameter of SatCom, which is gained through simulation, can be obtained with low computational complexity through the derived asymptotic results.

The remainder of this paper is organized as follows. We first describe the system and channel models of a single beam SatCom system under consideration in Section III In Section III] we derive the OP and SER performance in a single beam SatCom system. In Section IV] we explain the system and channel models of a multibeam SatCom system. In V, the OP and SER performance in a multibeam SatCom system are presented. Some simulation results and discussions are offered in Section VI. Finally, we conclude this paper in Section VII

\section{System and Channel Model of A Single Beam SATCOM SYSTEM}

First of all, we consider a SatCom downlink system where a satellite has a single beam as shown in Fig. 1. We assume users have a single antenna and are randomly distributed within the 


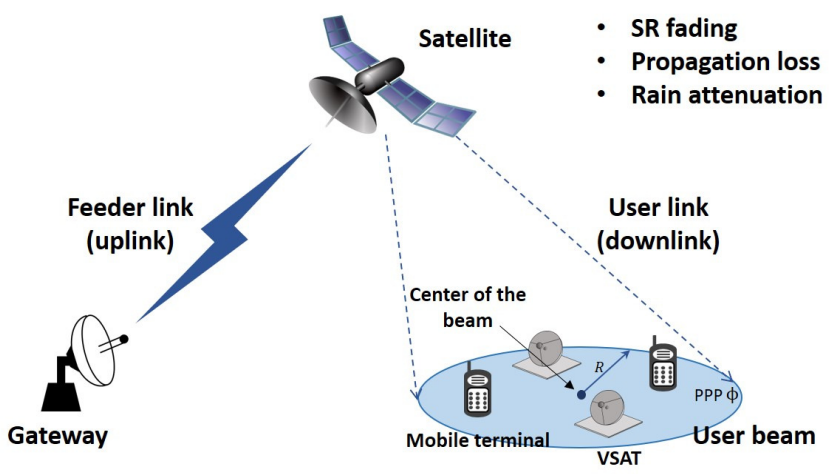

Fig. 1: System model of satellite communications with a single beam.

beam. We consider that their distribution follows a PPP $\Phi$, which has intensity $\rho$ in the closed disk of radius $R$. So, we note that the average number of users in a disk is $\rho \pi R^{2}$. When a single beam is employed, it can be a case of using one large beam or a multibeam situation with negligible inter-beam interference by using a high frequency reuse factor such as seven-frequency reuse. Since the signals from the satellite to users are mainly transmitted through Ka-band where the rain attenuation is a decisive factor [7], [9], [10], [37], the downlink signals in user link experience rain attenuation, beam gain, FSPL, and SR fading.

The channel coefficient $h_{k}$ from the satellite to the $k$ th user in the beam can be decomposed as

$$
h_{k}=A_{k} g_{k},
$$

where $A_{k}$ and $g_{k}$ denote link attenuation and small scale fading, respectively. The link attenuation is given by [38]

$$
A_{k}=s_{k} \frac{\lambda}{4 \pi d_{k}} G_{R} q_{k}
$$

where $s_{k}, \lambda$, and $G_{R}^{2}$ denote the rain attenuation coefficient, the carrier wavelength, and the user receiver antenna gain, respectively, and $d_{k}$ denotes the distance between the satellite and the $k$ th user. The satellite beam gain $q_{k}$ depending on the random user location can be approximated by [7]

$$
\begin{aligned}
q_{k} & =G_{\max } \alpha_{k}, \\
\alpha_{k} & =\left|\frac{J_{1}\left(u_{k}\right)}{2 u_{k}}+36 \frac{J_{3}\left(u_{k}\right)}{u_{k}^{3}}\right|,
\end{aligned}
$$

where $G_{\max }^{2}$ is the maximum satellite antenna gain. In (3), $J_{1}(\cdot)$ and $J_{3}(\cdot)$ are the first-kind Bessel functions of order 1 and 3 , respectively. Beam pattern $\alpha_{k}$ depends on the $k$ th user location. The variable $u_{k}$ is written as $u_{k}=$ $2.07123 \sin \left(\theta_{k}\right) / \sin \left(\theta_{3 \mathrm{~dB}}\right)$, where $\theta_{k}$ denotes the angle between the center of the beam and the $k$ th user, and $\theta_{3 \mathrm{~dB}}$ is the angle of the $3 \mathrm{~dB}$ power loss from the beam center. The satellite may not be positioned right above the center of the beam, but $\alpha_{k}$ can be assumed to be radially symmetric because the satellite's altitude is much higher than the distance between the beam center and the vertical lower point of the satellite. In fact, even if users are located at opposite edges of the same beam, the same beam gain values are obtained except for beams close to the polar regions.

The small scale fading $g_{k}$ is modeled as SR distribution whose LoS component and amplitudes of the scatter follow Nakagami- $m$ and Rayleigh distributions, respectively. SR fading is denoted as $\mathcal{S} \mathcal{R}\left(\Omega, b_{0}, m\right)$, where $\Omega$ is the average power of the LoS component, $2 b_{0}$ is the average power of the scatter components, and $m$ is the Nakagami parameter. We note that the SR fading is the most widely employed distribution modeling small scale fading in SatCom systems, and CDF of the SR fading power gain $g_{k}^{2}$ is given by [39]

$$
\begin{aligned}
F_{g_{k}^{2}}(x) & =\left(\frac{2 b_{0} m}{2 b_{0} m+\Omega}\right)^{m} \times \\
& \sum_{n=0}^{\infty} \frac{(m)_{n}}{n ! \Gamma(n+1)}\left(\frac{\Omega}{2 b_{0} m+\Omega}\right)^{n} \gamma\left(n+1, \frac{1}{2 b_{0}} x\right),
\end{aligned}
$$

where $\Gamma(\cdot)$ denotes the Gamma function, $\gamma(\cdot, \cdot)$ is the lower incomplete Gamma function, and $(a)_{n}$ is the Pochhammer symbol [40]. We suppose the users in the beam suffer from the independent and identically distributed (i.i.d.) SR fading [20], [36], [41].

Therefore, the received SNR of the $k$ th user in the beam can be written as

$$
\mathrm{SNR}_{k}=P\left(s_{k} \frac{\lambda G_{R} G_{\text {max }}}{4 \pi d_{k} \sqrt{\kappa T B}}\right)^{2} \alpha_{k}^{2} g_{k}^{2},
$$

where $P$ denotes the transmit power of the satellite, and we assume the noise variance of each user is the same. In (5), $\kappa, T$, and $B$ are the Boltzmann constant, the receiver noise temperature, and the bandwidth, respectively. Note that we also suppose that the feeder link is ideal, perfect CSI is available at gateway, and the Doppler spread is negligible.

\section{Performance Analysis in a Single Beam SATCOM SYSTEM}

Before analyzing the OP and SER performance, we obtain the CDF of $\mathrm{SNR}_{k}$. Due to the complicated form of $\alpha_{k}$ in (3b), it is very intractable to obtain the exact the probability density function (PDF) of $\alpha_{k}$ and, thus, we will utilize a stochastic geometry framework over random user location. However, since it is intractable to apply the obtained CDF to a stochastic geometry framework directly, we derive the asymptotic CDF using high SNR approximation, and take advantage of it to analyze the performance.

The exact CDF of the received SNR at the $k$ th user can be written as

$$
\begin{aligned}
F_{\mathrm{SNR}_{k}}\left(x \mid \alpha_{k}\right) & =\operatorname{Pr}\left(\mathrm{SNR}_{k} \leq x\right) \\
& =\operatorname{Pr}\left(g_{k}^{2} \leq \frac{x}{P A_{k}^{2}}\right)=F_{g_{k}^{2}}\left(\frac{x}{P A_{k}^{2}}\right) .
\end{aligned}
$$

With the high SNR approximation, CDF can be approximated as (7), where (a) holds that incomplete gamma function can be approximated at high SNR $(P \rightarrow \infty)$ [42]; in (b), we employ the confluent hypergeometric function ${ }_{1} F_{1}(\cdot ; \cdot ; \cdot)$ [40]; (c) holds that the confluent hypergeometric function goes to one at high SNR. The distance between the user and satellite 


$$
\begin{aligned}
F_{\mathrm{SNR}_{k}}\left(x \mid \alpha_{k}\right) & =\left(\frac{2 b_{0} m}{2 b_{0} m+\Omega}\right)^{m} \sum_{n=0}^{\infty} \frac{(m)_{n}}{n ! \Gamma(n+1)}\left(\frac{\Omega}{2 b_{0} m+\Omega}\right)^{n} \gamma\left(n+1, \frac{1}{2 b_{0}} \frac{x}{P A_{k}^{2}}\right) \\
& \stackrel{(a)}{\approx}\left(\frac{2 b_{0} m}{2 b_{0} m+\Omega}\right)^{m} \sum_{n=0}^{\infty} \frac{(m)_{n}}{n ! \Gamma(n+1)}\left(\frac{\Omega}{2 b_{0} m+\Omega}\right)^{n} \frac{1}{n+1}\left(\frac{1}{2 b_{0}} \frac{x}{P A_{k}^{2}}\right)^{n+1} \\
& \stackrel{(b)}{=}\left(\frac{2 b_{0} m}{2 b_{0} m+\Omega}\right)^{m}{ }_{1} F_{1}\left(m ; 2 ; \frac{\Omega}{2 b_{0} m+\Omega} \frac{1}{2 b_{0}} \frac{x}{P A_{k}^{2}}\right) \frac{1}{2 b_{0}} \frac{x}{P A_{k}^{2}} \\
& \approx\left(\frac{2 b_{0} m}{2 b_{0} m+\Omega}\right)^{m} \frac{1}{2 b_{0}} \frac{x}{P A_{k}^{2}} \\
& =\left(\frac{2 b_{0} m}{2 b_{0} m+\Omega}\right)^{m} \frac{1}{2 b_{0}} \frac{x}{P \alpha_{k}^{2}}\left(s_{k} \frac{\lambda G_{R} G_{\max }}{4 \pi d_{k} \sqrt{\kappa T B}}\right)^{-2}
\end{aligned}
$$

can be approximated to the height of the satellite (i.e., $d_{k}$ for all $k$ is constant.) because the altitude of the GEO satellite is very high compared to the distance between the center of the beam and users. For simplicity, even if the power gain due to rain attenuation in $\mathrm{dB}$ follows lognormal distribution, we assume users in the same beam suffer from the same rain attenuation (i.e., $s_{k}$ for all $k$ is constant.) since rain attenuation has spatial correlation in the tens of kilometers [7]. Accordingly, $\left(s_{k} \frac{\lambda G_{R} G_{\max }}{4 \pi d_{k} \sqrt{\kappa T B}}\right)^{2}$ can be substituted with a constant $D$. Thus, the asymptotic CDF of the received SNR at the $k$ th user can be written as

$$
F_{\mathrm{SNR}_{k}}^{\infty}\left(x \mid \alpha_{k}\right)=\left(\frac{2 b_{0} m}{2 b_{0} m+\Omega}\right)^{m} \frac{x}{2 b_{0} P D \alpha_{k}^{2}} .
$$

In the following subsections, we analyze OP and SER of the users distributed as PPP in the beam for three cases.

\section{A. Outage Probability}

When $\gamma$ is the SNR of a certain user in the beam, the OP of the user is defined as the probability that $\gamma$ falls below a predefined threshold $\gamma_{\text {th }}$, which can be expressed as

$$
P_{\text {out }}\left(\gamma_{\mathrm{th}}\right)=\operatorname{Pr}\left(\gamma \leq \gamma_{\mathrm{th}}\right)=F_{\gamma}\left(\gamma_{\mathrm{th}}\right),
$$

where $F_{\gamma}(\cdot)$ denotes the $\mathrm{CDF}$ of $\gamma$. We apply stochastic geometry to the asymptotic CDF so as to analyze OP in the cases of the user with the maximum received SNR, the user with the minimum received SNR, and the randomly selected user among the users within the beam. Since SatCom systems cover a very large area, there can be numerous users in the beam. In practical SatCom, users are scheduled based on their channel quality with time division multiplexing. For example of DVB-S2, the user terminals (receivers) estimate their channel qualities such as signal-to-noise-plus-interference ratio (SINR) and send it back to a gateway. The modulation and coding scheme corresponding to channel quality can be fed back to a gateway. The gateway selects the proper modulation and coding scheme of user terminals for each time instant. However, in practice, the data traffic can have different priority and queuing state at the buffers of gateway and it is not always chosen only by channel quality. It is very complicated to analyze the practical selection schemes along with traffic scheduling. Thus, most of the previous literature deals with the impact of user selection solely as follows. As in [7], only one user per beam can be served. In this case, the optimal performance can be obtained by using best link selection (BLS), which selects the user with the maximum received SNR for time-varying channels [43], or the fairness can be guaranteed by using random link selection (RLS) that randomly selects users [43], [44]. However, the latest SatCom standards consider multicast systems that serve multiple users simultaneously in each beam, such as [8], [10], [38]. For multicast systems, since the signals are transmitted according to the minimum received SINR of users receiving the same signal, it is appropriate to consider worst link selection (WLS).

1) BLS: In order to evaluate the best performance of the user link, we take account of the user that has the maximum received SNR in the beam. Among users that follow PPP $\Phi$, the maximum received SNR can be written as

$$
\gamma_{\max }=\max _{k \in \Phi} \mathrm{SNR}_{k} \text {. }
$$

There may not be even a single user in the beam as PPP. However, if there are zero users, it is always considered that outage has occurred, which results in the CDF not converging to zero or one. Hence, we rule out the case no users are generated when users are distributed in the beam with PPP. Therefore, the CDF of the maximum received SNR can be calculated as (11), where $\mathrm{E}_{\Phi}$ denotes expectation over $\Phi$. In (11), (a) holds for the independence of $\left\{\mathrm{SNR}_{k} ; k \in \Phi\right\}$ and (b) follows by using (6) and Poisson distribution. If we make use of the exact CDF of SNR and stochastic geometry, the expectation terms in (11) can be calculated as (12), where (a) follows from the probability generating functional lemma (PGFL) for PPP [45]; according to PPP, users are generated and uniformly distributed within the beam. The satellite beam pattern $\alpha_{k}$ depends on the user location. In the single beam situation, the beam pattern is determined by how far the user location is from the center of the beam because only the beam containing the user is considered without interference. Therefore, in (12), $\alpha_{k}$ can be replaced with $\alpha(r)$ when the user is $r$ away. In other words, $\alpha(r)$ can be written as

$$
\alpha(r)=\left|\frac{J_{1}(u(r))}{2 u(r)}+36 \frac{J_{3}(u(r))}{u^{3}(r)}\right|,
$$




$$
\begin{aligned}
F_{\gamma_{\max }}^{\mathrm{SNR}}(x) & =\operatorname{Pr}\left(\max _{k \in \Phi} \mathrm{SNR}_{k} \leq x \mid \Phi>0\right) \\
& =\frac{1}{\operatorname{Pr}(\Phi>0)} \times \operatorname{Pr}\left(\max _{k \in \Phi} \mathrm{SNR}_{k} \leq x, \Phi>0\right) \\
& =\frac{1}{1-\operatorname{Pr}(\Phi=0)} \times\left\{\operatorname{Pr}\left(\max _{k \in \Phi} \mathrm{SNR}_{k} \leq x\right)-\operatorname{Pr}\left(\max _{k \in \Phi} \mathrm{SNR}_{k} \leq x, \Phi=0\right)\right\} \\
& \stackrel{(a)}{=} \frac{1}{1-\operatorname{Pr}(\Phi=0)} \times\left\{\mathrm{E}_{\Phi}\left[\prod_{k \in \Phi} \operatorname{Pr}\left(\mathrm{SNR}_{k} \leq x\right)\right]-\operatorname{Pr}(\Phi=0)\right\} \\
& \stackrel{(b)}{=} \frac{1}{1-\exp \left(-\rho \pi R^{2}\right)} \times\left\{\mathrm{E}_{\Phi}\left[\prod_{k \in \Phi} F_{\mathrm{SNR}_{k}}\left(x \mid \alpha_{k}\right)\right]-\exp \left(-\rho \pi R^{2}\right)\right\}
\end{aligned}
$$

$$
\begin{aligned}
& \mathrm{E}_{\Phi}\left[\prod_{k \in \Phi} F_{\mathrm{SNR}_{k}}\left(x \mid \alpha_{k}\right)\right] \\
& =\mathrm{E}_{\Phi}\left[\prod_{k \in \Phi}\left(\frac{2 b_{0} m}{2 b_{0} m+\Omega}\right)^{m} \sum_{n=0}^{\infty} \frac{(m)_{n}}{n ! \Gamma(n+1)}\left(\frac{\Omega}{2 b_{0} m+\Omega}\right)^{n} \gamma\left(n+1, \frac{1}{2 b_{0}} \frac{x}{P D \alpha_{k}^{2}}\right)\right] \\
& \stackrel{(a)}{=} \exp \left[-\rho \int_{0}^{2 \pi} \int_{0}^{R} r\left\{1-\left(\frac{2 b_{0} m}{2 b_{0} m+\Omega}\right)^{m} \sum_{n=0}^{\infty} \frac{(m)_{n}}{n ! \Gamma(n+1)}\left(\frac{\Omega}{2 b_{0} m+\Omega}\right)^{n} \gamma\left(n+1, \frac{1}{2 b_{0}} \frac{x}{P D \alpha^{2}(r)}\right)\right\} d r d \phi\right]
\end{aligned}
$$

$$
\begin{aligned}
F_{\gamma_{\min }}^{\mathrm{SNR}}(x) & =\operatorname{Pr}\left(\min _{k \in \Phi} \operatorname{SNR}_{k} \leq x \mid \Phi>0\right) \\
& =1-\operatorname{Pr}\left(\min _{k \in \Phi} \operatorname{SNR}_{k}>x \mid \Phi>0\right) \\
& =1-\frac{1}{1-\operatorname{Pr}(\Phi=0)} \times\left\{\mathrm{E}_{\Phi}\left[\prod_{k \in \Phi}\left(1-\operatorname{Pr}\left(\operatorname{SNR}_{k} \leq x\right)\right)\right]-\operatorname{Pr}(\Phi=0)\right\} \\
& =1-\frac{1}{1-\exp \left(-\rho \pi R^{2}\right)} \times\left\{\mathrm{E}_{\Phi}\left[\prod_{k \in \Phi}\left(1-F_{\mathrm{SNR}_{k}}\left(x \mid \alpha_{k}\right)\right)\right]-\exp \left(-\rho \pi R^{2}\right)\right\}
\end{aligned}
$$

where $u(r)$ depends on the angle $\theta$ between users and center of the beam as seen in Section [I] When $H$ denotes the height of the satellite, $\sin (\theta)=r / \sqrt{r^{2}+H^{2}}$. As such, when using the exact CDF of SNR (i.e., evaluating (11) with (12)), it is very challenging to obtain the result in a closed form by integrating the composite function consisting of trigonometric function, Bessel function, and incomplete gamma function 1 Instead, we plug the asymptotic CDF into the expectation term in (11) and apply PGFL again as in 12 , which can be evaluated as

$$
\begin{aligned}
& \mathrm{E}_{\Phi}\left[\prod_{k \in \Phi} F_{\mathrm{SNR}_{k}}^{\infty}\left(x \mid \alpha_{k}\right)\right] \\
& =\mathrm{E}_{\Phi}\left[\prod_{k \in \Phi}\left(\frac{2 b_{0} m}{2 b_{0} m+\Omega}\right)^{m} \frac{1}{2 b_{0}} \frac{x}{P D \alpha_{k}^{2}}\right] \\
& =\exp \left(-\rho \pi R^{2}\right) \times
\end{aligned}
$$

${ }^{1}$ We note that the exact analytical results can be numerically obtained and verified with simulations later.

$$
\exp [\rho 2 \pi\left(\frac{2 b_{0} m}{2 b_{0} m+\Omega}\right)^{m} \frac{1}{2 b_{0}} \frac{x}{P D} \underbrace{\int_{0}^{R} \frac{r}{\alpha^{2}(r)} d r}_{C_{R, 1}}],
$$

where an integral term $C_{R, 1}$ remains, but it can be regarded as a constant if the beam size is determined and computed once. Hence, the asymptotic CDF of the maximum received SNR can be written as

$$
\begin{aligned}
F_{\gamma_{\max }}^{\mathrm{SNR}, \infty}(x) & =\frac{\exp \left(-\rho \pi R^{2}\right)}{1-\exp \left(-\rho \pi R^{2}\right)} \times \\
& \left\{\exp \left[\rho 2 \pi\left(\frac{2 b_{0} m}{2 b_{0} m+\Omega}\right)^{m} \frac{x}{2 b_{0} P D} C_{R, 1}\right]-1\right\},
\end{aligned}
$$

where we can approximate the asymptotic CDF by first-order Taylor linearization at 0 as

$$
\begin{aligned}
F_{\gamma_{\max }}^{\mathrm{SNR}, \infty,(1)}(x) & =\frac{\exp \left(-\rho \pi R^{2}\right)}{1-\exp \left(-\rho \pi R^{2}\right)} \\
& \times\left\{\rho 2 \pi\left(\frac{2 b_{0} m}{2 b_{0} m+\Omega}\right)^{m} \frac{x}{2 b_{0} P D} C_{R, 1}\right\} .
\end{aligned}
$$


Therefore, the OP for BLS scheme in SatCom can be computed as $P_{\text {out }}^{\mathrm{BLS}, \infty}=F_{\gamma_{\max }}^{\infty}\left(\gamma_{\mathrm{th}}\right)$.

2) WLS: In the multicast channel, we consider the user that has the minimum received SNR within the beam to provide the same information at the minimum achievable rate to the multiple users under guaranteed performance. The minimum received SNR can be expressed as

$$
\gamma_{\min }=\min _{k \in \Phi} \mathrm{SNR}_{k}
$$

As in a similar way to the BLS scheme, the CDF of the minimum received SNR can be derived as (18). The exact CDF of the minimum received SNR can be derived by employing the exact CDF of SNR with PGFL, but it is quite difficult to deal with for the same reason as in (12). Thus, we calculate it with the asymptotic CDF as in BLS. The asymptotic CDF of the minimum received SNR can be obtained as

$$
\begin{aligned}
F_{\gamma_{\text {min }}}^{\mathrm{SNR}, \infty}(x) & =\frac{1}{1-\exp \left(-\rho \pi R^{2}\right)} \times \\
& \left\{1-\exp \left[-\rho 2 \pi\left(\frac{2 b_{0} m}{2 b_{0} m+\Omega}\right)^{m} \frac{x}{2 b_{0} P D} C_{R, 1}\right]\right\},
\end{aligned}
$$

where we can also approximate the asymptotic CDF by firstorder Taylor linearization at 0 as

$$
\begin{aligned}
& F_{\gamma_{\min }}^{\mathrm{SNR}, \infty,(1)}(x)=\frac{1}{1-\exp \left(\rho \pi R^{2}\right)} \times \\
& \left\{-\rho 2 \pi\left(\frac{2 b_{0} m}{2 b_{0} m+\Omega}\right)^{m} \frac{x}{2 b_{0} P D} C_{R, 1}\right\} \text {. }
\end{aligned}
$$

3) RLS: To achieve fairness of users in the beam and compare the performance to the previous two cases, we obtain the CDF of the SNR of the user that is selected at random. Since choosing one user randomly among the several users within the beam is equivalent to the circumstance where only one user is arbitrarily located in the beam, we make use of the latter for the mathematical tractability. Thus, the SNR of the randomly chosen user can be written as

$$
\gamma_{\text {random }}=\mathrm{SNR}_{k},
$$

where $\mathrm{SNR}_{k}$ denotes the SNR of an arbitrarily chosen user. Since a single user is uniformly distributed within the beam, the CDF of random received SNR can be calculated as

$$
\begin{aligned}
F_{\gamma_{\text {random }}}^{\mathrm{SNR}}(x) & =\operatorname{Pr}\left(\mathrm{SNR}_{k} \leq x \mid \Phi=1\right) \\
& =\int_{0}^{2 \pi} \int_{0}^{R} \frac{r}{\pi R^{2}} F_{\mathrm{SNR}_{k}}\left(x \mid \alpha_{k}\right) d r d \phi .
\end{aligned}
$$

We also employ the asymptotic CDF of SNR for the same reason as the preceding two cases, and the asymptotic CDF can be obtained as

$$
F_{\gamma_{\text {random }}}^{\mathrm{SNR}, \infty}(x)=\left(\frac{2 b_{0} m}{2 b_{0} m+\Omega}\right)^{m} \frac{x}{b_{0} P D R^{2}} C_{R, 1} .
$$

In the next subsection, we obtain the moment generating function (MGF) to calculate the SER for each case using the obtained CDF.

\section{B. Symbol Error Rate}

The SER of $M$-ary phase shift keying ( $M$-PSK) modulation can be written as [46]

$$
P_{M-\mathrm{PSK}}=\frac{1}{\pi} \int_{0}^{\pi-\frac{\pi}{M}} M_{\gamma}\left(-\frac{\sin ^{2}(\pi / M)}{\sin ^{2} \theta}\right) d \theta,
$$

where $M_{\gamma}(s)=\mathrm{E}\left[e^{s \gamma}\right]$ is the MGF of $\gamma$. The single integral form of 24 can be further approximated as an alternative closed-form which is given by [16], [36], [47]

$$
\begin{aligned}
P_{M-\mathrm{PSK}} \approx & \left(\frac{\theta_{M}}{2 \pi}-\frac{1}{6}\right) M_{\gamma}\left(-\sin ^{2}\left(\frac{\pi}{M}\right)\right)+\frac{1}{4} M_{\gamma}\left(-\frac{4}{3} \sin ^{2}\left(\frac{\pi}{M}\right)\right) \\
& +\left(\frac{\theta_{M}}{2 \pi}-\frac{1}{4}\right) M_{\gamma}\left(-\frac{\sin ^{2}(\pi / M)}{\sin ^{2} \theta_{M}}\right),
\end{aligned}
$$

where $\theta_{M}=\pi-\pi / M$. To analyze the SER of the user for each case, we derive the MGF expression for each case with the asymptotic CDF calculated above.

1) BLS: By taking the derivative of (15), the PDF of the maximum SNR can be expressed as

$$
f_{\gamma_{\max }}^{\mathrm{SNR}, \infty}(x)=\frac{V W_{1}}{1-V} \exp \left(W_{1} x\right),
$$

where, for the sake of convenience, $V$ and $W_{1}$ denote

$$
\begin{aligned}
V & =\exp \left(-\rho \pi R^{2}\right) \\
W_{1} & =\rho 2 \pi\left(\frac{2 b_{0} m}{2 b_{0} m+\Omega}\right)^{m} \frac{1}{2 b_{0} P D} C_{R, 1} .
\end{aligned}
$$

With (26), the MGF of the maximum received SNR can be calculated as

$$
M_{\gamma_{\max }}^{\mathrm{SNR}, \infty}(s)=-\frac{V W_{1}}{(1-V)\left(s+W_{1}\right)},
$$

where this follows from $s+W_{1}<0$, which is satisfied because $s$ is negative for SER of $M$-PSK and $W_{1}$ approaches zero at high SNR.

2) WLS: From (19), the PDF of the minimum SNR can be written as

$$
f_{\gamma_{\min }}^{\mathrm{SNR}, \infty}(x)=\frac{W_{1}}{1-V} \exp \left(-W_{1} x\right) .
$$

The MGF of the minimum SNR can be computed as

$$
M_{\gamma_{\min }}^{\mathrm{SNR}, \infty}(s)=-\frac{W_{1}}{(1-V)\left(s-W_{1}\right)},
$$

where this holds for $s-W_{1}<0$, which is also satisfied because of $W_{1}>0$ and $s<0$ for SER of the $M$-PSK.

3) RLS: With 23, the PDF of the SNR selected randomly can be written as

$$
f_{\gamma_{\text {random }}}^{\mathrm{SNR} \infty}(x)=\frac{W_{1}}{\rho \pi R^{2}},
$$

where the asymptotic PDF is a constant function. The MGF of the randomly selected user can be obtained as

$$
M_{\gamma_{\mathrm{random}}}^{\mathrm{SNR}, \infty}(s)=-\frac{W_{1}}{\rho \pi R^{2} s},
$$

where this holds for $s<0$, which is always satisfied for SER of the $M$-PSK. 


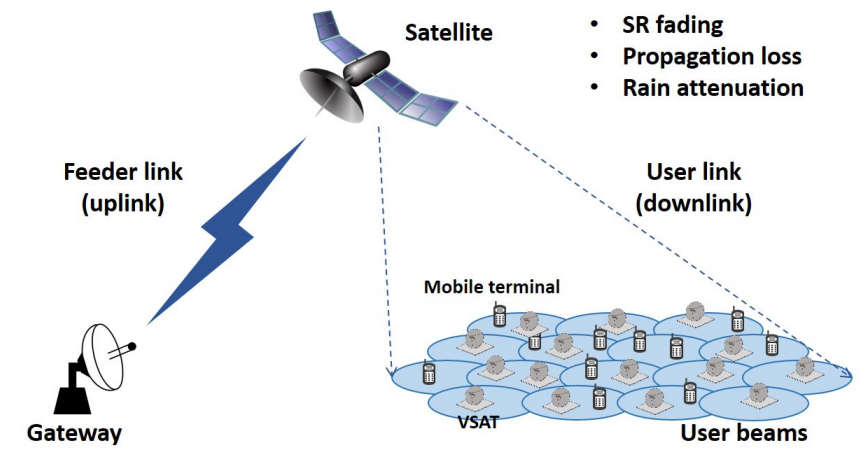

Fig. 2: System model of satellite communications with multiple beams.

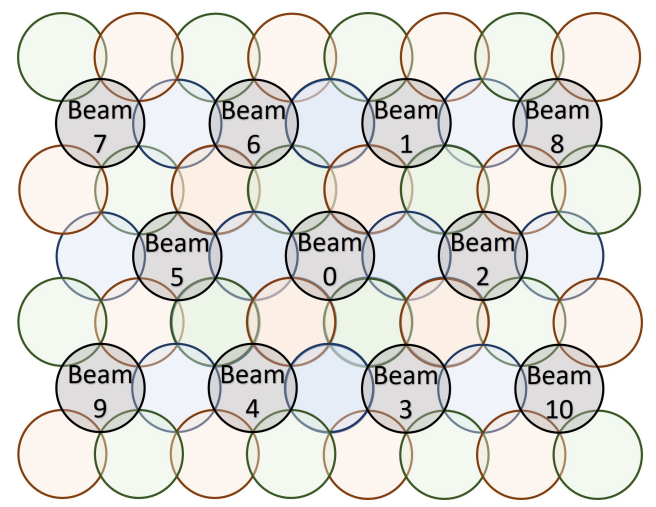

Fig. 3: Four-frequency reuse pattern: Beams with the same color use the same frequency band. When beam- 0 is the reference beam, beam- 1 to beam- 6 are the first tier of the reference beam.

\section{System and Channel Model of A Multibeam SATCOM SYSTEM}

In this section, we consider a SatCom downlink system exploiting multiple beams as shown in Fig. 2. Unlike the single beam situation, since several beams are operational on the same frequency band in a multibeam situation, the beams undergo inter-beam interference from surrounding beams. We assume that the beam of interest is the 0th beam among the multiple beams and the users following the distribution of PPP $\Phi$ in the beam go through inter-beam interference from the $L$ beams. In other words, a set of beams is denoted as $\mathcal{L}=\{0,1, \cdots, L\}$, in which $l=0$ represents the beam of interest and $1 \leq l \leq L$ mean the beams that interfere with the 0th beam.

For example, the four-frequency reuse pattern is shown in Fig. 3, where the beams in the same color use the same frequency band. When beam- 0 is the beam of interest, beam- 1 to beam-10 are the beams that interfere with beam- 0 . Since the distance from beam- $i(i=7,8,9,10)$ to beam- 0 is farther than the distance from beam- $j(j=1,2, \cdots, 6)$ to beam- 0 , interference from beam- $i$ can be negligible.

The channel model defined in Section III is used for multibeam SatCom system. However, inter-beam interference should be taken into consideration in multibeam SatCom system. Therefore, when $h_{k, l}$ is the channel coefficient from the $l$ th beam of the satellite to the $k$ th user in the 0th beam, the received SINR of the $k$ th user in the beam can be written as

$$
\begin{aligned}
\operatorname{SINR}_{k} & =\frac{P\left|h_{k, 0}\right|^{2}}{P \sum_{l=1}^{L}\left|h_{k, l}\right|^{2}+1} \\
& =\frac{P\left(s_{k} \frac{\lambda G_{R} G_{\max }}{4 \pi d_{k} \sqrt{\kappa T B}}\right)^{2} \alpha_{k, 0}^{2} g_{k, 0}^{2}}{P \sum_{l=1}^{L}\left(s_{k} \frac{\lambda G_{R} G_{\max }}{4 \pi d_{k} \sqrt{\kappa T B}}\right)^{2} \alpha_{k, l}^{2} g_{k, l}^{2}+1} \\
& =\frac{P D \alpha_{k, 0}^{2} g_{k, 0}^{2}}{P D \sum_{l=1}^{L} \alpha_{k, l}^{2} g_{k, l}^{2}+1},
\end{aligned}
$$

where $q_{k, l}=G_{\max } \alpha_{k, l}$ and $g_{k, l}$ stand for satellite beam gain and SR fading from the $l$ th beam, respectively.

\section{Performance Analysis in a Multibeam Satcom SYSTEM}

As in Section III], we obtain the CDF of $\operatorname{SINR}_{k}$ before we derive CDF and MGF for three user selection schemes. The exact CDF of the received SINR at the $k$ th user can be evaluated as 35, where $f_{g_{k, l}^{2}}(\cdot)$ denotes the PDF of $g_{k, l}^{2}$, which is given as [48]

$$
\begin{array}{r}
f_{g_{k, l}^{2}}\left(y_{l}\right)=\left(\frac{2 b_{0} m}{2 b_{0} m+\Omega}\right)^{m} \frac{1}{2 b_{0}} \exp \left(-\frac{y_{l}}{2 b_{0}}\right) \times \\
{ }_{1} F_{1}\left(m ; 1 ; \frac{\Omega}{2 b_{0}\left(2 b_{0} m+\Omega\right)} y_{l}\right) .
\end{array}
$$

It is difficult to obtain the closed form of (35) since it includes several special functions in the multiple integral forms. Even though it can be solved in a closed form, applying stochastic geometry to that form is unmanageable. As seen in Section IIII $\alpha_{k, l}$ is a composite function of the Bessel function depending on the user's position. Hence, we approximate (35) using (8) with [40, Eq.(7.621)], and the asymptotic CDF of the received SINR at the $k$ th user can be derived as 37), where ${ }_{2} F_{1}(\cdot, \cdot ; \cdot ; \cdot)$ denotes Gauss hypergeometric function [40].

In the following subsections, we investigate OP and SER in the multibeam situation by applying stochastic geometry. As in the single beam situation, we attain CDF and derive MGF to analyze the performance for three cases such as BLS, WLS, and RLS.

\section{A. $B L S$}

The maximum received SINR among users in the 0th beam is expressed as

$$
\gamma_{\max }=\max _{k \in \Phi} \operatorname{SINR}_{k}
$$

As (11), when only one or more users are distributed, the $\mathrm{CDF}$ of maximum received SINR can be derived as 39, where we need to calculate the expectation term to obtain the CDF of maximum received SINR with PGFL as (40). In (40), $\alpha_{l}(r, \phi)$ is a function of the position of the $l$ th beam and the users, which is given as 


$$
\begin{aligned}
& F_{\mathrm{SINR}_{k}}\left(x \mid \alpha_{k, 0}, \alpha_{k, 1}, \cdots, \alpha_{k, L}\right) \\
& =\operatorname{Pr}\left(\operatorname{SINR}_{k} \leq x\right) \\
& =\operatorname{Pr}\left(g_{k, 0}^{2} \leq \frac{P D \sum_{l=1}^{L} \alpha_{k, l}^{2} g_{k, l}^{2}+1}{P D \alpha_{k, 0}^{2}} x\right) \\
& =\int_{0}^{\infty} \cdots \int_{0}^{\infty} \operatorname{Pr}\left(g_{k, 0}^{2} \leq \frac{P D \sum_{l=1}^{L} \alpha_{k, l}^{2} g_{k, l}^{2}+1}{P D \alpha_{k, 0}^{2}} x \mid g_{k, l}^{2}=y_{l}, l=1, \cdots, L\right) f_{g_{k, 1}^{2}, \cdots, g_{k, L}^{2}}\left(y_{1}, \cdots, y_{L}\right) d y_{1} \cdots d y_{L} \\
& =\int_{0}^{\infty} \cdots \int_{0}^{\infty} F_{g_{k, 0}^{2}}\left(\frac{P D \sum_{l=1}^{L} \alpha_{k, l}^{2} y_{l}+1}{P D \alpha_{k, 0}^{2}} x\right) f_{g_{k, 1}^{2}}\left(y_{1}\right) \cdots f_{g_{k, L}^{2}}\left(y_{L}\right) d y_{1} \cdots d y_{L}
\end{aligned}
$$

$$
\begin{aligned}
F_{\mathrm{SINR}_{k}}^{\infty}\left(x \mid \alpha_{k, 0}, \alpha_{k, 1}, \cdots, \alpha_{k, L}\right) & =\int_{0}^{\infty} \cdots \int_{0}^{\infty} F_{g_{k, 0}^{2}}^{\infty}\left(\frac{\sum_{l}^{L} D^{2} \alpha_{k, l}^{2} y_{l}+1}{P D^{2} \alpha_{k, 0}^{2} g_{k, 0}^{2}} x\right) f_{P g_{k, 1}^{2}}\left(y_{1}\right) \cdots f_{P g_{k, L}^{2}}\left(y_{L}\right) d y_{1} \cdots d y_{L} \\
& =\left(\left(\frac{2 b_{0} m}{2 b_{0} m+\Omega}\right)^{m} \frac{1}{2 b_{0} P D} \frac{1}{\alpha_{k, 0}^{2}}+\left(\frac{2 b_{0} m}{2 b_{0} m+\Omega}\right)^{2 m}{ }_{2} F_{1}\left(m, 2 ; 1 ; \frac{\Omega}{2 b_{0} m+\Omega}\right) \frac{1}{\alpha_{k, 0}^{2}} \sum_{l=1}^{L} \alpha_{k, l}^{2}\right) x
\end{aligned}
$$

$$
\begin{aligned}
F_{\gamma_{\max }}^{\mathrm{SINR}}(x) & =\operatorname{Pr}\left(\max _{k \in \Phi} \operatorname{SINR}_{k} \leq x \mid \Phi>0\right) \\
& =\frac{1}{\operatorname{Pr}(\Phi>0)} \times \operatorname{Pr}\left(\max _{k \in \Phi} \operatorname{SINR}_{k} \leq x, \Phi>0\right) \\
& =\frac{1}{1-\operatorname{Pr}(\Phi=0)} \times\left\{\operatorname{Pr}\left(\max _{k \in \Phi} \operatorname{SINR}_{k} \leq x\right)-\operatorname{Pr}\left(\max _{k \in \Phi} \operatorname{SINR}_{k} \leq x, \Phi=0\right)\right\} \\
& =\frac{1}{1-\operatorname{Pr}(\Phi=0)} \times\left\{\mathrm{E}_{\Phi}\left[\prod_{k \in \Phi} \operatorname{Pr}\left(\operatorname{SINR}_{k} \leq x\right)\right]-\operatorname{Pr}(\Phi=0)\right\} \\
& =\frac{1}{1-\exp \left(-\rho \pi R^{2}\right)} \times\left\{\mathrm{E}_{\Phi}\left[\prod_{k \in \Phi} F_{\operatorname{SINR}_{k}}\left(x \mid \alpha_{k, 0}, \alpha_{k, 1}, \cdots, \alpha_{k, L}\right)\right]-\exp \left(-\rho \pi R^{2}\right)\right\}
\end{aligned}
$$

$$
\begin{aligned}
& \mathrm{E}_{\Phi}\left[\prod_{k \in \Phi} F_{\mathrm{SINR}_{k}}\left(x \mid \alpha_{k, 0}, \alpha_{k, 1}, \cdots, \alpha_{k, L}\right)\right] \\
& =\mathrm{E}_{\Phi}\left[\prod_{k \in \Phi} \int_{0}^{\infty} \cdots \int_{0}^{\infty} F_{g_{k, 0}^{2}}\left(\frac{P D \sum_{l=1}^{L} \alpha_{k, l}^{2} y_{l}+1}{P D \alpha_{k, 0}^{2}} x\right) f_{g_{k, 1}^{2}}\left(y_{1}\right) \cdots f_{g_{k, L}^{2}}\left(y_{L}\right) d y_{1} \cdots d y_{L}\right] \\
& =\exp \left[-\rho \int_{0}^{2 \pi} \int_{0}^{R} r\left\{1-\int_{0}^{\infty} \cdots \int_{0}^{\infty} F_{g_{0}^{2}}\left(\frac{P D \sum_{l=1}^{L} \alpha_{l}^{2}(r, \phi) y_{l}+1}{P D \alpha_{0}^{2}(r)} x\right) f_{g_{1}^{2}}\left(y_{1}\right) \cdots f_{g_{L}^{2}}\left(y_{L}\right) d y_{1} \cdots d y_{L}\right\} d r d \phi\right]
\end{aligned}
$$

$$
F_{\gamma_{\max }}^{\mathrm{SINR}, \infty}(x)=\frac{\exp \left(-\rho \pi R^{2}\right)}{1-\exp \left(-\rho \pi R^{2}\right)} \times\left\{\exp \left[\rho x\left(2 \pi\left(\frac{2 b_{0} m}{2 b_{0} m+\Omega}\right)^{m}+\left(\frac{2 b_{0} m}{2 b_{0} m+\Omega}\right)^{2 m}{ }_{2} F_{1}\left(m, 2 ; 1 ; \frac{\Omega}{2 b_{0} m+\Omega}\right) C_{R, 2}\right)\right]-1\right\}
$$

$$
\begin{aligned}
F_{\gamma_{\min }}^{\mathrm{SINR}}(x) & =\operatorname{Pr}\left(\min _{k \in \Phi} \operatorname{SINR}_{k} \leq x \mid \Phi>0\right) \\
& =1-\frac{1}{1-\exp \left(-\rho \pi R^{2}\right)} \times\left\{\mathrm{E}_{\Phi}\left[\prod_{k \in \Phi}\left(1-F_{\mathrm{SINR}_{k}}\left(x \mid \alpha_{k, 0}, \alpha_{k, 1}, \cdots, \alpha_{k, L}\right)\right)\right]-\exp \left(-\rho \pi R^{2}\right)\right\}
\end{aligned}
$$




$$
\alpha_{l}(r, \phi)=\left|\frac{J_{1}\left(u_{l}(r, \phi)\right)}{2 u_{l}(r, \phi)}+36 \frac{J_{3}\left(u_{l}(r, \phi)\right)}{u_{l}^{3}(r, \phi)}\right|,
$$

where $u_{l}(r, \phi)$ depends on the angle between the center of $l$ th beam and users in 0th beam. Note that $\alpha_{0}(r)$ is the same as (13). As mentioned above, when applying PGFL to the exact $\mathrm{CDF}$, the closed form cannot be obtained. Therefore, plugging the asymptotic CDF in (37) into (40), we derive the asymptotic $\mathrm{CDF}$ of the maximum received SINR as [42), where $C_{R, 2}$ denotes

$$
C_{R, 2}=\int_{0}^{2 \pi} \int_{0}^{R} \frac{r}{\alpha_{0}^{2}(r)} \sum_{l=1}^{L} \alpha_{l}^{2}(r, \phi) d r d \phi .
$$

We can see the integral terms in (42), e.g., $C_{R, 1}$ and $C_{R, 2}$ as constant values computed once if the beam size and the number of interference beams are set. Then, we can obtain the OP of the maximum received SINR with (42).

Using (42), the MGF of maximum received SINR can be expressed as

$$
M_{\gamma_{\max }}^{\mathrm{SINR, \infty}}(s) \stackrel{(a)}{=}-\frac{V W_{2}}{(1-V)\left(s+W_{2}\right)},
$$

where $W_{2}$ denotes

$$
\begin{aligned}
W_{2}= & \rho\left(2 \pi\left(\frac{2 b_{0} m}{2 b_{0} m+\Omega}\right)^{m} \frac{1}{2 b_{0} P D} C_{R, 1}+\right. \\
& \left.\left(\frac{2 b_{0} m}{2 b_{0} m+\Omega}\right)^{2 m}{ }_{2} F_{1}\left(m, 2 ; 1 ; \frac{\Omega}{2 b_{0} m+\Omega}\right) C_{R, 2}\right),
\end{aligned}
$$

and (a) holds for $s+W_{2}<0$, which is satisfied in the high power regime. Hence, we can obtain the SER of $M$-PSK by calculating 25, with 44.

\section{B. WLS}

The minimum received SINR can be written as

$$
\gamma_{\min }=\min _{k \in \Phi} \operatorname{SINR}_{k} .
$$

From (18), the CDF of minimum received SINR can be obtained as (47). For the same reason as BLS, we obtain the asymptotic CDF of minimum received SINR employing 37) as follows

$$
F_{\gamma_{\min }}^{\mathrm{SINR}, \infty}(x)=\frac{1}{1-V}\left(1-\exp \left(-W_{2} x\right)\right) .
$$

Therefore, the MGF of the minimum received SINR can be derived as

$$
M_{\gamma_{\min }}^{\mathrm{SINR}, \infty}(s)=-\frac{W_{2}}{(1-V)\left(s-W_{2}\right)},
$$

which holds for $s-W_{2}<0$. Because of $W_{2}>0$ and $s<0$ for SER of the $M$-PSK, it is satisfied.

\section{C. $R L S$}

As in the single beam situation, the SINR of a randomly selected user can be obtained when there is only one user. The received SINR of a randomly selected user can be expressed as

$$
\gamma_{\text {random }}=\operatorname{SINR}_{k}
$$

TABLE I: Parameters of the SR fading [48]

\begin{tabular}{|c|c|c|c|}
\hline Shadowing scenario & $\Omega$ & $b_{0}$ & $m$ \\
\hline Frequent heavy shadowing & $8.97 \times 10^{-4}$ & 0.063 & 0.739 \\
\hline Infrequent light shadowing & 1.29 & 0.158 & 19.4 \\
\hline Average shadowing & 0.835 & 0.126 & 10.1 \\
\hline
\end{tabular}

TABLE II: System Parameters [10]

\begin{tabular}{c|c}
\hline PARAMETER & VALUE \\
\hline \hline Satellite height & $35786 \mathrm{~km}(\mathrm{GEO})$ \\
\hline Link frequency band & $f_{c}=20 \mathrm{GHz}(\mathrm{Ka})$ \\
\hline Beam bandwidth & $B=500 \mathrm{MHz}$ \\
\hline Noise temperature & $T=517 \mathrm{~K}$ \\
\hline Boltzmann constant & $\kappa=1.3807 \times 10^{-23}$ \\
\hline Beam diameter & $250 \mathrm{~km}$ \\
\hline User antenna gain & $41.7 \mathrm{dBi}$ \\
\hline Satellite antenna gain & $52 \mathrm{dBi}$ \\
\hline 3 dB angle & $0.4^{\circ}$ \\
\hline Average rain attenuation & $-3.125 \mathrm{~dB}$ \\
\hline Outage threshold & $\gamma_{\mathrm{th}}=-2.85 \mathrm{~dB}$ \\
\hline
\end{tabular}

where $\operatorname{SINR}_{k}$ denotes the SINR of a single user that is uniformly distributed in the 0th beam. Hence, the asymptotic CDF of the RLS received SINR can be derived as

$$
F_{\gamma_{\text {random }}}^{\mathrm{SINR} \infty}(x)=\frac{W_{2}}{\rho \pi R^{2}} x .
$$

Accordingly, the MGF of the received SINR can be obtained as

$$
M_{\gamma_{\text {random }}}^{\mathrm{SINR}, \infty}(s)=-\frac{W_{2}}{\rho \pi R^{2} s},
$$

which holds for $s<0$. Thus, it is always satisfied for SER of the $M$-PSK.

\section{Numerical Results}

In this section, we present numerical results to confirm the validity of the exact analysis on OP and SER and demonstrate the accuracy of the obtained asymptotic expressions at high power regime. In the simulation, we exploit SR fading parameters given in Table 1] [48], and set the intensity of the PPP to $\rho=\left[4.0744 \times 10^{-11}, 1.0186 \times 10^{-10}\right]$ which means there are two users and five users on average within a beam for each value of $\rho$, respectively, i.e., $\rho \pi R^{2}=[2,5]$. The system parameters used in the simulations are listed in Table II] [10], where $\gamma_{\mathrm{th}}=-2.85 \mathrm{~dB}$ is the value that provides the error-free communication with the most robust modulation and coding rate for the normal frame length [49]. Since the power per beam is practically more than $20 \mathrm{dBW}$, the figures hereafter are plotted to include larger values [9], [11], [50]. In the single case, we assume a situation in which interference is ignored by using high frequency reuse. In the multibeam case, there are several methods, but the four-color frequency is dealt with as in [1], [50]. Therefore, the beams of the same size are used for equivalent comparison of user performance in two situations. When exploiting the four-color frequency reuse, we assume that users experience interference from only the first tier that is the closest six beams to the reference beam and the number of interfering beams is six, i.e., $L=6$. Furthermore, we choose the beam that is formed vertically below the satellite. Since the beam pattern depends on the distance from the beam center 


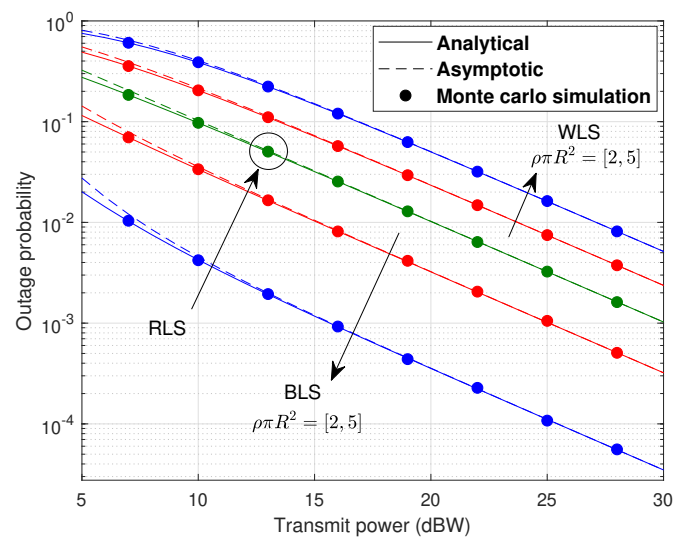

(a)

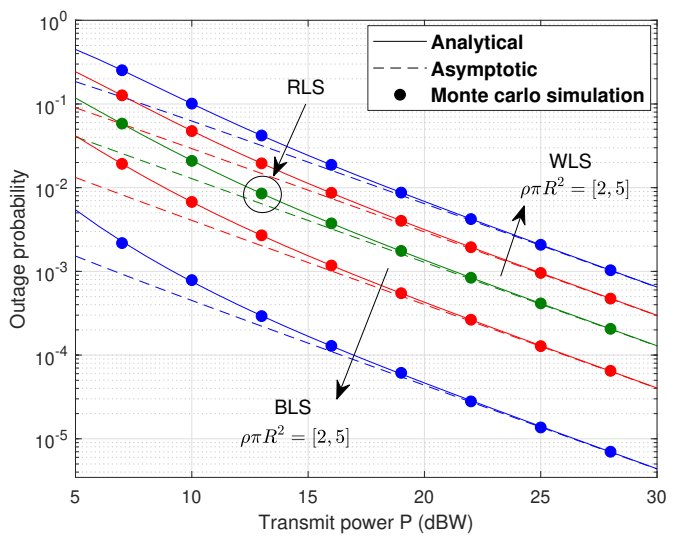

(b)

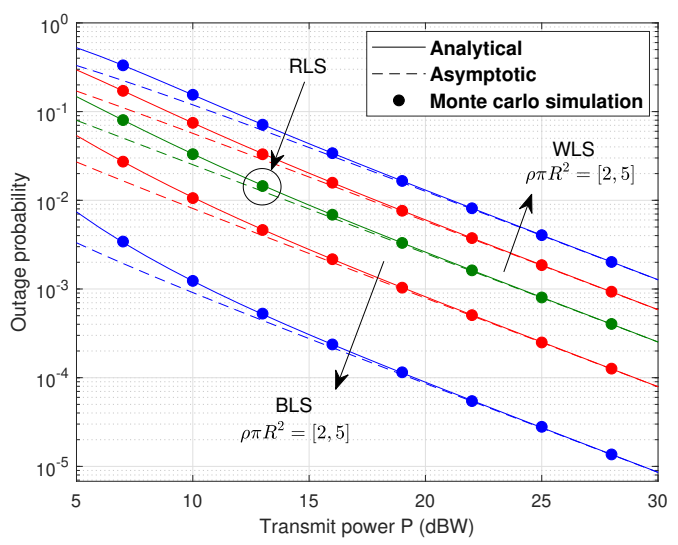

(c)

Fig. 4: OP versus the transmit power under different shadowing models in single beam situation: (a) frequent heavy shadowing, (b) infrequent light shadowing, and (c) average shadowing.

regardless of the beam's position owing to the satellite's very high altitude, which beam is selected has no effect on the result. In other words, even if any beam at a different location were selected, the same simulation results would be obtained because it could not affect the beam pattern.

Fig. 4 shows the OP versus transmit power for various SR

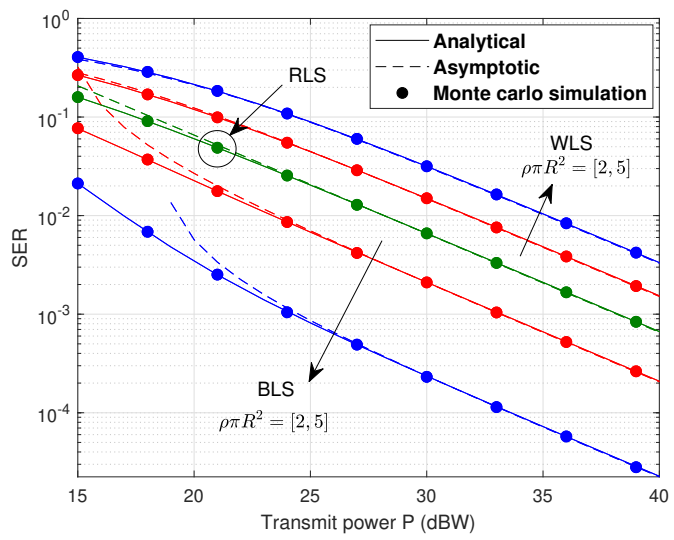

(a)

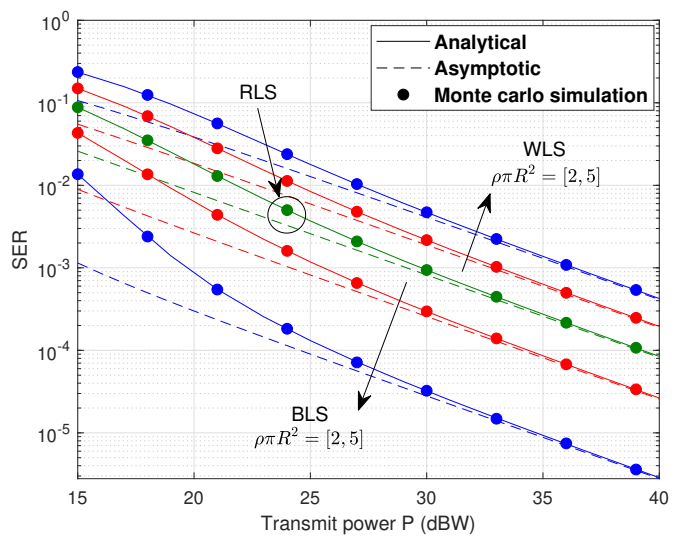

(b)

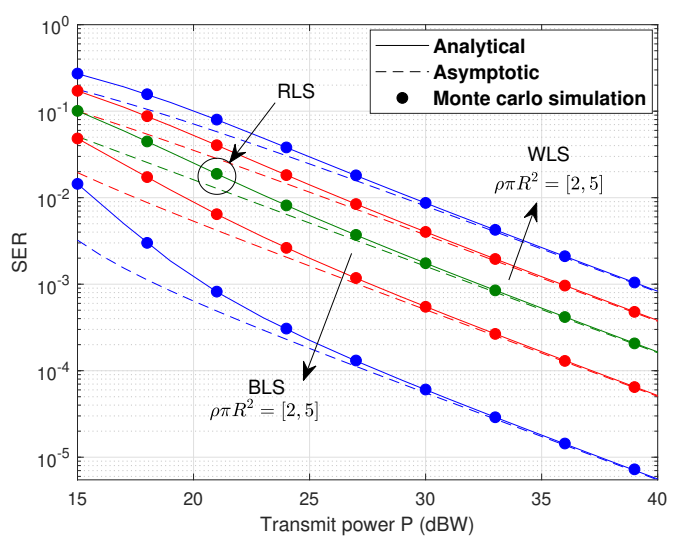

(c)

Fig. 5: SER versus the transmit power under different shadowing models in single beam situation: (a) frequent heavy shadowing, (b) infrequent light shadowing, and (c) average shadowing.

fading scenarios in the single beam situation. We obtain the exact expressions by truncating the infinite series, i.e., $n=20$ and calculate their simulation results. As we expect, the exact results agree well with Monte Carlo simulation results, and the asymptotic curves match well with them at high SNR, which implies that the theoretical analysis accurately evaluates the 


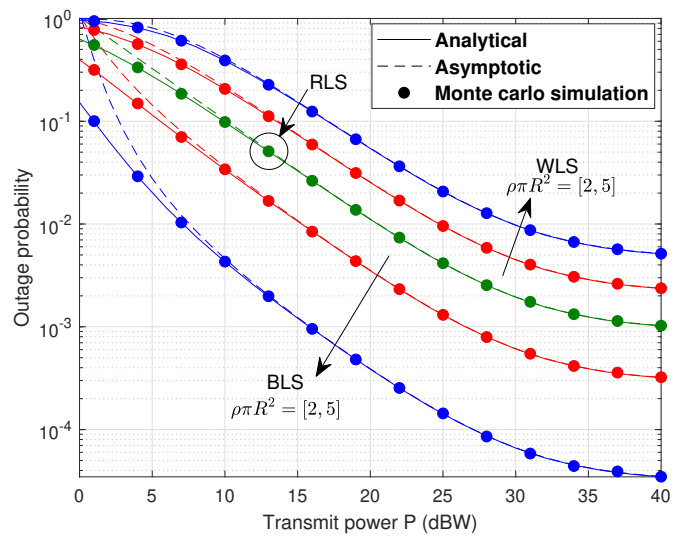

(a)

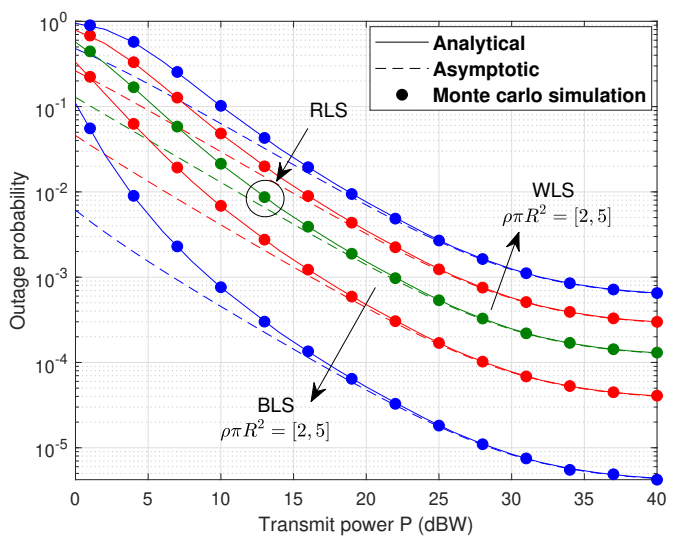

(b)

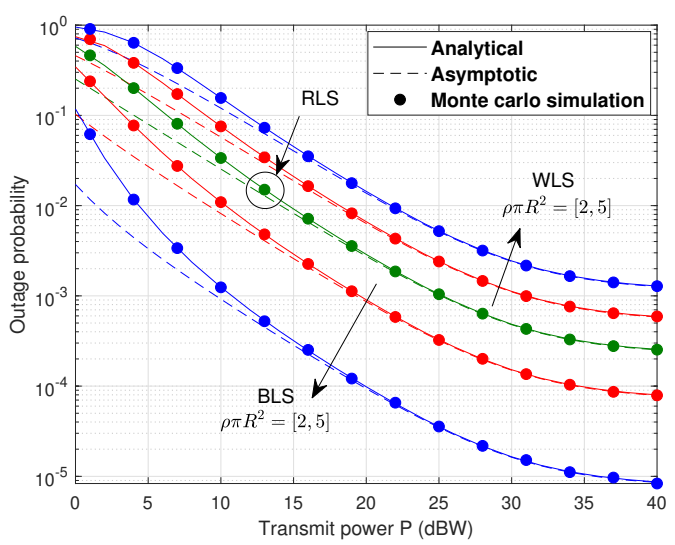

(c)

Fig. 6: OP versus the transmit power under different shadowing models in multibeam situation: (a) frequent heavy shadowing, (b) infrequent light shadowing, and (c) average shadowing.

OP. Moreover, we can confirm the gap in power gain between different user densities, while there is no change in diversity order.

Fig. 5 depicts the SER performances for 8-PSK modulation in different SR fading scenarios. As in the simulation of the OP, we obtain the simulation results and confirm that

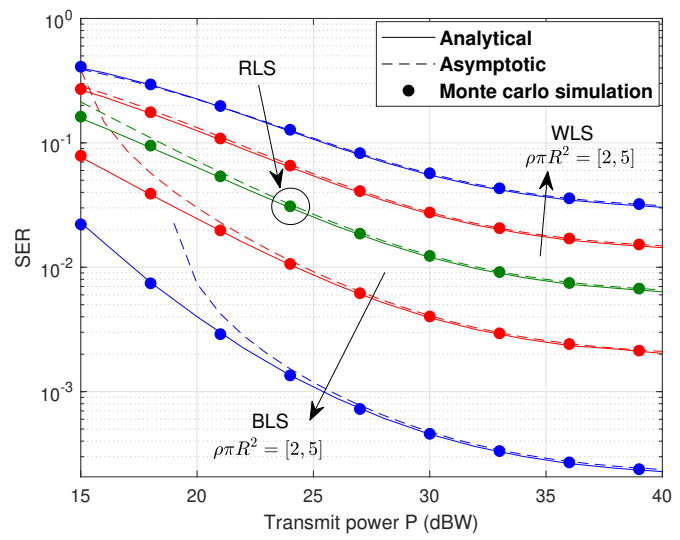

(a)

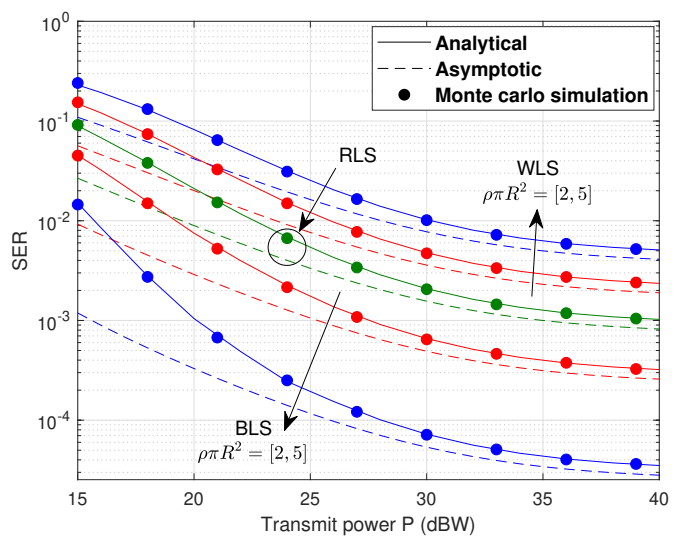

(b)

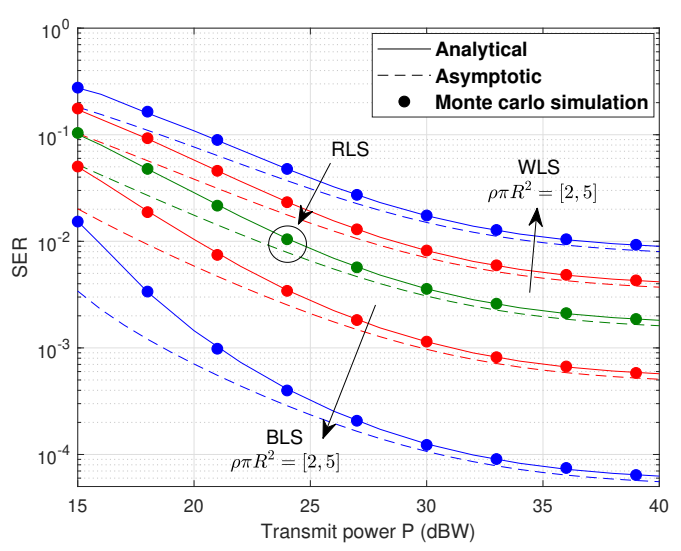

(c)

Fig. 7: SER versus the transmit power under different shadowing models in multibeam situation: (a) frequent heavy shadowing, (b) infrequent light shadowing, and (c) average shadowing.

the asymptotic results fit well with the results of the exact expressions at high SNR. Similar to the case of the OP, it can be seen that the better the channel scenario, the more slowly the asymptotic curves are closer to the results of the exact forms. In the process of obtaining MGF using the BLS scheme, the condition $s+W_{1}<0$ is required. When calculating 


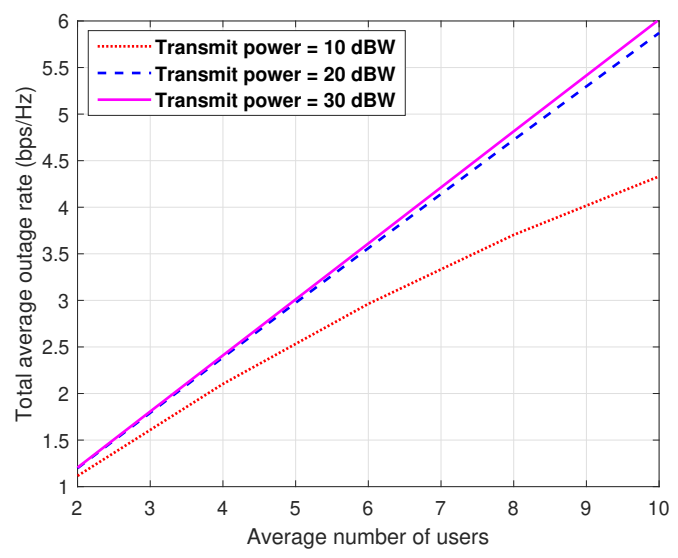

(a)

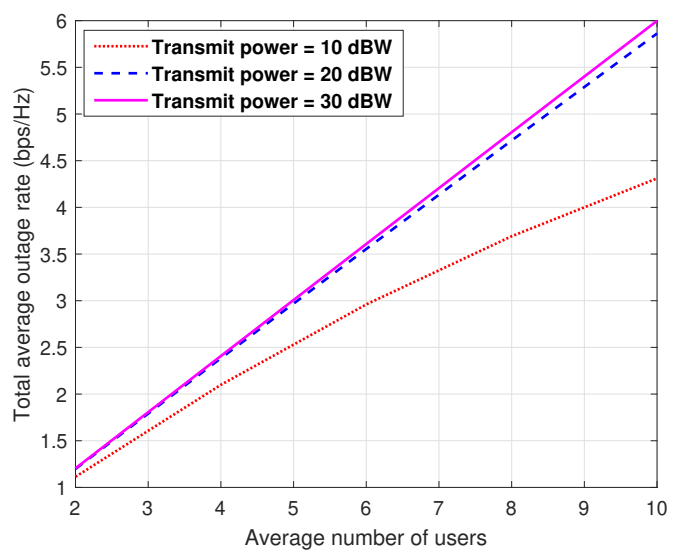

(b)

Fig. 8: Total average throughput versus the average number of users over average shadowing: (a) single beam situation, and (b) multibeam situation.

the SER, $s$ is always negative, but $W_{1}$ is always positive. In the lower power regime, the condition may not be satisfied, which results in uncomputed parts. However, the closer to the high power regime, $W_{1}$ approaches 0 and the SER can be calculated.

Figs. 6 and 7 present the performances in multibeam situation. In Fig. 6, the OP performances are illustrated for different SR fading scenarios. As in the single beam situation, the exact results match precisely with Monte Carlo simulation results, and the asymptotic curves very tightly follow them at high power regime. The SER performances for 8-PSK modulation are plotted in Fig. 7. We can see that the curves of exact forms match well with the simulation results and the asymptotic curves are very accurate. In particular, it is more accurate at lower SER. As in Fig. 5, when obtaining the MGF using the BLS scheme in multibeam situation, the condition $s+W_{2}<0$ is required. Due to this condition, the calculation of the SER with the BLS scheme may not be performed in the lower power regime.

Fig. 8 plots the average throughput of all users in a multicast SatCom system. As shown in Fig. 4 (or 6), in the case of selecting user that has minimum received SNR (or
SINR), the OP increases as the average number of users in a beam increases. This means that as the number of users that receive the signal from the satellite increases, the number of users with outage also increases. Hence, it is interesting to see the total average throughput of users in the beam, which denotes correctly received average rate of all users. The average throughput of a user can be expressed as $R_{o}=\left(1-P_{\text {out }}\left(\gamma_{\mathrm{th}}\right)\right) \log _{2}\left(1+\gamma_{\mathrm{th}}\right)[51]$. Although the number of users having outage increases with the average number of users in the received SNR (or SINR) in the WLS scheme, we can find out that the satellite can cover more users and the total average throughput (i.e., $\rho \pi R^{2} R_{o}$ ) increases.

Fig. 9 shows the OP versus $3 \mathrm{~dB}$ beam angle according to the user selection schemes in a multibeam situation and presents the three situations where the beam radius is different and the power per beam is set to $20 \mathrm{dBW}$. In a multibeam situation, due to inter-beam interference, the optimal $3 \mathrm{~dB}$ angle to be applied in the satellite antenna differs according to the radius. Regardless of the user selection scheme, the same optimal $3 \mathrm{~dB}$ angle is obtained at the same radius, and a larger angle is required as the radius increases. Since the simulation results and the asymptotic results agree well at the optimum angle, the optimum $3 \mathrm{~dB}$ angle can be obtained from the asymptotic results, which reduces the computational complexity.

\section{CONCLUSION}

In this paper, we analyzed the performance of downlink users which are distributed as PPP within each beam considering FSPL, beam pattern, and SR fading as random factors in a SatCom system. By taking advantage of stochastic geometry, we derived the analytic expressions and asymptotic closed forms of the OP and SER. Numerical results demonstrated that the analytic and simulation results show a reasonably good match and the asymptotic results converge to the analytic results at high power regime.

\section{ACKNOWLEDGMENT}

The authors would like to thank the anonymous reviewers and editor Prof. Harpreet S. Dhillon for valuable comments and feedback which improved the quality of the paper.

\section{REFERENCES}

[1] M. A. Vazquez, A. Perez-Neira, D. Christopoulos, S. Chatzinotas, B. Ottersten, P. Arapoglou, A. Ginesi, and G. Tarocco, "Precoding in multibeam satellite communications: Present and future challenges," IEEE Wireless Commun., vol. 23, no. 6, pp. 88-95, Dec. 2016.

[2] G. Giambene, S. Kota, and P. Pillai, "Satellite-5G integration: A network perspective," IEEE Netw., vol. 32, no. 5, pp. 25-31, Sep. 2018.

[3] S. Dang, O. Amin, B. Shihada, and M.-S. Alouini, "What should 6G be?" Nat. Electron., vol. 3, no. 1, pp. 20-29, Jan. 2020.

[4] E. Yaacoub and M. Alouini, "A key 6G challenge and opportunity-Connecting the base of the pyramid: A survey on rural connectivity," Proc. IEEE, vol. 108, no. 4, pp. 533-582, Apr. 2020.

[5] M. Giordani and M. Zorzi, "Non-terrestrial networks in the $6 \mathrm{G}$ era: Challenges and opportunities," IEEE Netw., vol. 35, no. 2, pp. 244-251, Mar. 2021.

[6] R. T. Schwarz, T. Delamotte, K. Storek, and A. Knopp, "MIMO applications for multibeam satellites," IEEE Trans. Broadcast., vol. 65 , no. 4, pp. 664-681, Dec. 2019. 


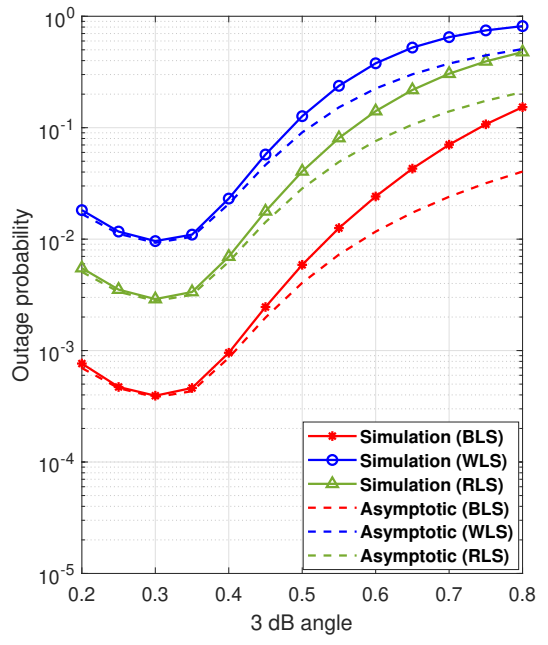

(a)

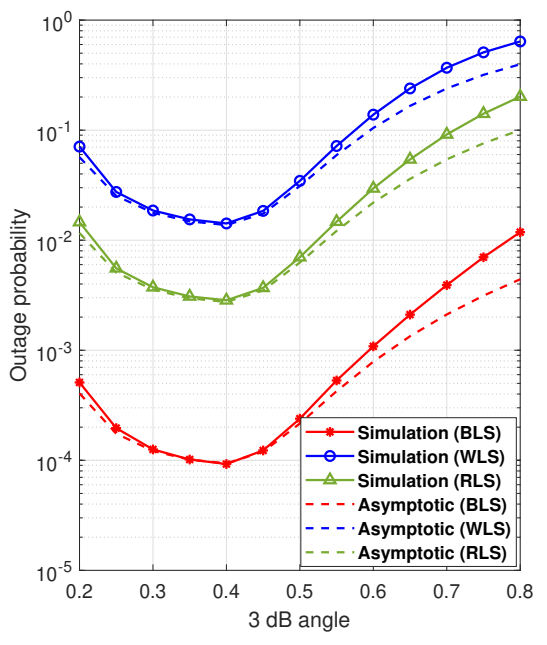

(b)

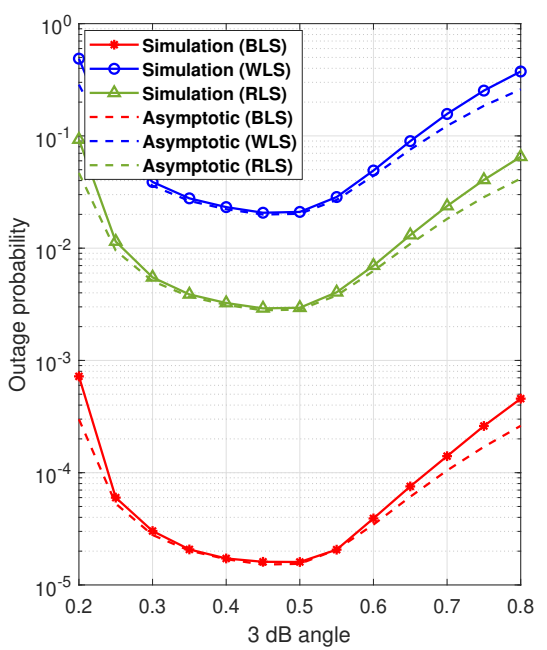

(c)

Fig. 9: OP versus $3 \mathrm{~dB}$ beam angle under different user selection schemes: (a) $R=100 \mathrm{~km}$, (b) $R=125 \mathrm{~km}$, and (c) $R=150$ $\mathrm{km}$.

[7] G. Zheng, S. Chatzinotas, and B. Ottersten, "Generic optimization of linear precoding in multibeam satellite systems," IEEE Trans. Wireless Commun., vol. 11, no. 6, pp. 2308-2320, Jun. 2012.

[8] D. Christopoulos, S. Chatzinotas, and B. Ottersten, "Multicast multigroup precoding and user scheduling for frame-based satellite communications," IEEE Trans. Wireless Commun., vol. 14, no. 9, pp. 4695-4707, Sep 2015.

[9] V. Joroughi, M. Á. Vázquez, and A. I. Pérez-Neira, "Precoding in multigateway multibeam satellite systems," IEEE Trans. Wireless Commun., vol. 15, no. 7, pp. 4944-4956, Jul. 2016.

[10] J. Wang, L. Zhou, K. Yang, X. Wang, and Y. Liu, "Multicast precoding for multigateway multibeam satellite systems with feeder link interference," IEEE Trans. Wireless Commun., vol. 18, no. 3, pp. 1637-1650, Mar. 2019.

[11] S. Chatzinotas, G. Zheng, and B. Ottersten, "Energy-efficient MMSE beamforming and power allocation in multibeam satellite systems," in Proc. 2011 Conference Record of the Forty Fifth Asilomar Conference on Signals, Systems and Computers (ASILOMAR), Pacific Grove, CA, USA, Nov. 2011, pp. 1081-1085.

[12] C. Qi and X. Wang, "Precoding design for energy efficiency of multibeam satellite communications," IEEE Commun. Lett., vol. 22, no. 9, pp. 1826-1829, Sep. 2018.

[13] Y. Ruan, Y. Li, C. Wang, R. Zhang, and H. Zhang, "Energy efficient power allocation for delay constrained cognitive satellite terrestrial networks under interference constraints," IEEE Trans. Wireless Commun., vol. 18, no. 10, pp. 4957-4969, Oct. 2019.

[14] V. Joroughi, E. Lagunas, S. Andrenacci, N. Maturo, S. Chatzinotas, J. Grotz, and B. Ottersten, "Deploying joint beam hopping and precoding in multibeam satellite networks with time variant traffic," in Proc. Global Conf. Signal Inf. Process. (GlobalSIP), Anaheim, CA, USA, Nov. 2018, pp. 1081-1085.

[15] M. G. Kibria, E. Lagunas, N. Maturo, D. Spano, and S. Chatzinotas, "Precoded cluster hopping in multi-beam high throughput satellite systems," in Proc. IEEE Global Communications Conference (GLOBECOM), Waikoloa, HI, USA, Dec. 2019, pp. 1-6.

[16] M. R. Bhatnagar and A. M.K., "Performance analysis of AF based hybrid satellite-terrestrial cooperative network over generalized fading channels," IEEE Commun. Lett., vol. 17, no. 10, pp. 1912-1915, Oct. 2013.

[17] A. M.K. and M. R. Bhatnagar, "Beamforming and combining in hybrid satellite-terrestrial cooperative systems," IEEE Commun. Lett., vol. 18, no. 3, pp. 483-486, Mar. 2014

[18] L. Yang and M. O. Hasna, "Performance analysis of amplify-andforward hybrid satellite-terrestrial networks with cochannel interference," IEEE Trans. Commun., vol. 63, no. 12, pp. 5052-5061, Dec. 2015.

[19] A. M. K., "Channel estimation and detection in hybrid satel- lite-terrestrial communication systems," IEEE Trans. Veh. Technol., vol. 65 , no. 7, pp. 5764-5771, Jul. 2016.

[20] K. Guo, M. Lin, B. Zhang, W. Zhu, J. Wang, and T. A. Tsiftsis, "On the performance of LMS communication with hardware impairments and interference," IEEE Trans. Commun., vol. 67, no. 2, pp. 1490-1505, Feb. 2019.

[21] P. K. Sharma, P. K. Upadhyay, D. B. da Costa, P. S. Bithas, and A. G. Kanatas, "Performance analysis of overlay spectrum sharing in hybrid satellite-terrestrial systems with secondary network selection," IEEE Trans. Wireless Commun., vol. 16, no. 10, pp. 6586-6601, Oct 2017.

[22] K. An, M. Lin, T. Liang, J. Wang, J. Wang, Y. Huang, and A. L. Swindlehurst, "Performance analysis of multi-antenna hybrid satelliteterrestrial relay networks in the presence of interference," IEEE Trans. Commun., vol. 63, no. 11, pp. 4390-4404, Nov. 2015.

[23] P. K. Upadhyay and P. K. Sharma, "Max-max user-relay selection scheme in multiuser and multirelay hybrid satellite-terrestrial relay systems," IEEE Commun. Lett., vol. 20, no. 2, pp. 268-271, Feb. 2016.

[24] Z. Lin, M. Lin, W. Zhu, J. Wang, and J. Cheng, "Robust secure beamforming for wireless powered cognitive satellite-terrestrial networks," IEEE Trans. Cognit. Commun. Netw., early access, pp. 1-1, Aug. 2020.

[25] O. Y. Kolawole, S. Vuppala, M. Sellathurai, and T. Ratnarajah, "On the performance of cognitive satellite-terrestrial networks," IEEE Trans. Cognit. Commun. Netw., vol. 3, no. 4, pp. 668-683, Dec. 2017.

[26] T. Bai and R. W. Heath, "Coverage and rate analysis for millimeter-wave cellular networks," IEEE Trans. Wireless Commun., vol. 14, no. 2, pp. $1100-1114$, Feb. 2015.

[27] H. S. Dhillon, R. K. Ganti, F. Baccelli, and J. G. Andrews, "Modeling and analysis of K-tier downlink heterogeneous cellular networks," IEEE J. Sel. Areas Commun., vol. 30, no. 3, pp. 550-560, Apr. 2012.

[28] H. S. Dhillon and J. G. Andrews, "Downlink rate distribution in heterogeneous cellular networks under generalized cell selection," IEEE Wireless Commun. Lett., vol. 3, no. 1, pp. 42-45, Feb. 2014.

[29] H. ElSawy, A. Sultan-Salem, M.-S. Alouini, and M. Z. Win, "Modeling and analysis of cellular networks using stochastic geometry: A tutorial," IEEE Commun. Surv. Tutor, vol. 19, no. 1, pp. 167-203, 1st Quart. 2017.

[30] V. V. Chetlur and H. S. Dhillon, "Downlink coverage analysis for a finite 3-D wireless network of unmanned aerial vehicles," IEEE Trans. Commun., vol. 65, no. 10, pp. 4543-4558, Oct. 2017.

[31] E. Turgut and M. C. Gursoy, "Downlink analysis in unmanned aerial vehicle (UAV) assisted cellular networks with clustered users," IEEE Access, vol. 6, pp. 36313-36324, May 2018.

[32] T. A. Khan and M. Afshang, "A stochastic geometry approach to doppler characterization in a LEO satellite network," in Proc.2020 IEEE International Conference on Communications (ICC), Dublin, Ireland, Jun. 2020, pp. 1-6. 
[33] M. Caus, M. A. Vázquez, and A. Pérez-Neira, "NOMA and interference limited satellite scenarios," in Proc. 2016 50th Asilomar Conference on Signals, Systems and Computers, Pacific Grove, CA, USA, Nov. 2016, pp. 497-501.

[34] A. I. Perez-Neira, M. Caus, M. A. Vazquez, and N. Alagha, "NOMA schemes for multibeam satellite communications," arXiv preprint arXiv: 1810.08440 , Oct. 2018

[35] X. Yan, H. Xiao, K. An, G. Zheng, and W. Tao, "Hybrid satellite terrestrial relay networks with cooperative non-orthogonal multiple access," IEEE Commun. Lett., vol. 22, no. 5, pp. 978-981, May 2018.

[36] X. Yan, H. Xiao, C. Wang, K. An, A. T. Chronopoulos, and G. Zheng, "Performance analysis of NOMA-based land mobile satellite networks," IEEE Access, vol. 6, pp. 31327-31339, Jun. 2018.

[37] M. Lin, Z. Lin, W. Zhu, and J. Wang, "Joint beamforming for secure communication in cognitive satellite terrestrial networks," IEEE J. Sel. Areas Commun., vol. 36, no. 5, pp. 1017-1029, May 2018.

[38] V. Joroughi, M. Á. Vázquez, and A. I. Pérez-Neira, "Generalized multicast multibeam precoding for satellite communications," IEEE Trans. Wireless Commun., vol. 16, no. 2, pp. 952-966, Feb. 2017.

[39] G. Alfano and A. D. Maio, "Sum of squared shadowed-Rice random variables and its application to communication systems performance prediction," IEEE Trans. Wireless Commun., vol. 6, no. 10, pp. 35403545, Oct. 2007.

[40] I. S. Gradshteyn and I. M. Ryzhik, Table of integrals, series, and products. Academic press, 2014.

[41] M. R. Bhatnagar and A. M.K., "On the closed-form performance analysis of maximal ratio combining in shadowed-Rician fading LMS channels," IEEE Commun. Lett., vol. 18, no. 1, pp. 54-57, Jan. 2014.

[42] Z. Ding, R. Schober, and H. V. Poor, "A general MIMO framework for NOMA downlink and uplink transmission based on signal alignment," IEEE Trans. Wireless Commun., vol. 15, no. 6, pp. 4438-4454, Jun. 2016.

[43] M. Sellathurai, S. Vuppala, and T. Ratnarajah, "User selection for multibeam satellite channels: A stochastic geometry perspective," in Proc. 2016 50th Asilomar Conference on Signals, Systems and Computers, Pacific Grove, CA, USA, Nov. 2016, pp. 487-491.

[44] A. Gharanjik, M. R. Bhavani Shankar, P. Arapoglou, M. Bengtsson, and B. Ottersten, "Precoding design and user selection for multibeam satellite channels," in Proc. 2015 IEEE 16th International Workshop on Signal Processing Advances in Wireless Communications (SPAWC), Stockholm, Sweden, Jun. 2015, pp. 420-424.

[45] M. Haenggi, Stochastic Geometry for Wireless Networks. Cambridge University Press, 2012.

[46] M. K. Simon and M.-S. Alouini, Digital communication over fading channels. John Wiley \& Sons, 2005, vol. 95.

[47] M. R. Mckay, A. Zanella, I. B. Collings, and M. Chiani, "Error probability and SINR analysis of optimum combining in Rician fading," IEEE Trans. Commun., vol. 57, no. 3, pp. 676-687, Mar. 2009.

[48] A. Abdi, W. C. Lau, M.-S. Alouini, and M. Kaveh, "A new simple model for land mobile satellite channels: First- and second-order statistics," IEEE Trans. Wireless Commun., vol. 2, no. 3, pp. 519-528, May 2003.

[49] Digital Video Broadcasting (DVB); Second Generation Framing Structure, Channel Coding and Modulation Systems for Broadcasting, Interactive Services, News Gathering and Other Broadband Satellite Applications. Part 2: DVB-S2 Extensions (DVB-S2X). ETSI EN 302 307-2 V1.1.1, European Broadcasting Union, Feb. 2015.

[50] Digital Video Broadcasting (DVB); Implementation guidelines for the second generation system for Broadcasting, Interactive Services, News Gathering and Other Broadband Satellite Applications. Part 2: S2 Extensions (DVB-S2X). ETSI TR 102 376-2 V1.1.1, European Broadcasting Union, Nov. 2015.

[51] A. Goldsmith, Wireless communications. Cambridge University Press, 2005.

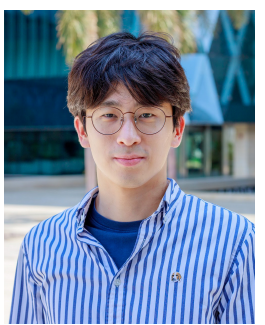

Dong-Hyoun Na (S'21) received the B.S. and M.S. degrees in electrical engineering from Korea University, Seoul, South Korea, in 2017 and 2019, respectively, where he is currently pursuing the Ph.D. degree with the School of Electrical Engineering. He is also pursuing the Ph.D. degree with the Division of Computer, Electrical, Mathematical Science and Engineering (CEMSE), King Abdullah University of Science and Technology (KAUST), Thuwal, Saudi Arabia. His current research interests include performance analysis and optimization of satellite communication systems.

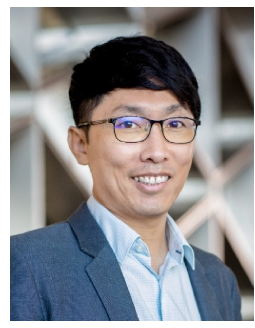

Ki-Hong Park (S'06-M'11-SM'19) received the B.Sc. degree in electrical, electronic, and radio engineering from Korea University, Seoul, South Korea, in 2005, and the joint M.S. and Ph.D. degrees from the School of Electrical Engineering, Korea University, in 2011. He joined the King Abdullah University of Science and Technology (KAUST), Thuwal, Saudi Arabia, in 2011, as a Post-Doctoral Fellow. Since 2014, he has been a Research Scientist of electrical and computer engineering with the Division of Computer, Electrical, Mathematical Science and Engineering (CEMSE), KAUST. His research interests include communication theory and its application to the design and performance evaluation of wireless communication systems and networks. His research interests include the application to optical wireless communications, nonterrestrial communications, and physical layer secrecy.

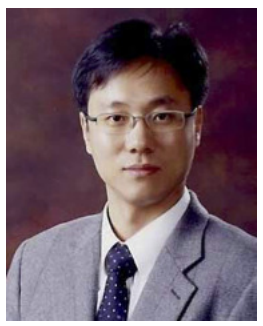

Young-Chai Ko (S'97-M'01-SM'06) received the B.Sc. degree in electrical and telecommunication engineering from the Hanyang University, Seoul, Korea and the M.S.E.E. and Ph.D. degrees in electrical engineering from the University of Minnesota, Minneapolis, MN in 1999 and 2001, respectively. He was with Novatel Wireless as a research scientist from January 2001 to March 2001. In March 2001, he joined the Texas Instruments, Inc., wireless center, San Diego, CA, as a senior engineer. He is now with the school of electrical engineering at Korea university as a professor. His current research interests include the design and evaluations of multi-user cellular system, MODEM architecture, mm-wave and $\mathrm{Tera} \mathrm{Hz}$ wireless systems.

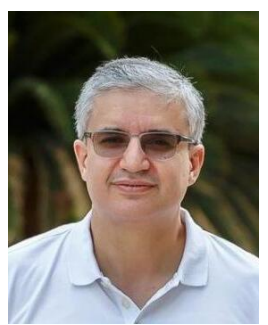

Mohamed-Slim Alouini (S'94-M'98-SM'03-F'09) was born in Tunis, Tunisia. He received the Ph.D. degree in Electrical Engineering from the California Institute of Technology (Caltech), Pasadena, CA, USA, in 1998. He served as a faculty member at the University of Minnesota, Minneapolis, MN, USA. then in the Texas A \& M University at Qatar, Education City, Doha, Qatar before joining King Abdullah University of Science and Technology (KAUST), Thuwal, Makkah Province, Saudi Arabia as a Professor of Electrical Engineering in 2009. His current research interests include modeling, design, and performance analysis of wireless communication systems. 